\title{
Application of Levenberg-Marguardt Algorithm for Prime Radio Propagation Wave Attenuation Modelling in Typical Urban, Suburban and Rural Terrains
}

\author{
Joseph Isabona \\ Department of Physics, Faculty of Science, Federal University Lokoja, PMB. 1154, Lokoja, Kogi State \\ E-mail: josabone@yahoo.com, joseph.isabona@ fulkoja.edu.ng \\ Divine O. Ojuh \\ Department of Physical Sciences, Faculty of Sciences, Benson Idahosa University, Benin City, Edo State \\ E-mail: dojuh@biu.edu.ng
}

Received: 19 March 2021; Accepted: 01 May 2021; Published: 08 June 2021

\begin{abstract}
The desire to achieve an adaptive prognostics regression learning processes of physical and empirical phenomenon is a complex task and open problem in radio frequency telecommunication engineering. One key method to solving such complex task or problems is by means of numerical based optimisation algorithms. The LevenbergMarquardt algorithm (LMA) is an efficient nonlinear parametric machine learning based modelling algorithm with optimal, fast, and accurate convergence speed. This paper proposes and demonstrates the real-time application of the LMA in developing a log-distance like propagation loss model based on received radio strength measurements conducted over deployed long term evolution (LTE) eNodeBs antennas in three different propagation areas. The LTE eNodeB signal propagation areas were selected to reflect typical urban, suburban and rural terrains which represent urban, suburban and rural terrains. The heights of the three eNodeBs are 30, 28 and $32 \mathrm{~m}$ respectively and each operate at $2.6 \mathrm{GHz}$ carrier frequency with $10 \mathrm{MHz}$ channel bandwidths. The resultant outcome of the proposed propagation loss modelling using LMA indicates a high approximation efficacy over the popular Gauss-Newton algorithm (GNA) modelling method, which has been used to benchmark the process. Precisely, the developed propagation loss model using LMA method attained lower maximum absolute error (MABE) of 7.73, 14.57and 10.53 for urban, suburban and rural terrains compared to the ones developed by GNA which yielded 15.19, 16.59 and 13.05 MABE values. The improved approximation performance of the LMA over the GNA can be ascribed to its capacity handle multiple free parameters and attain optimum solution irrespective of the selected values of initial guess parameters.
\end{abstract}

Index Terms: Propagation terrains, signal strength, Parametric modelling, Propagation loss, Gauss-Newton algorithm, ITU-R P.525 model, initial guess parameters, Levenberg-Marquardt algorithm.

\section{Introduction}

Over past two to three decades, the rate of different telecommunications system networks development and deployments has been at an incredible speed. This has be complemented by higher demands and proliferation of diverse portable multimedia communication devices by large number subscribers, globally.

The need to constantly provide good service quality at the user equipment devices and support telecommunications system networks deployments in different environments, proper modeling of the propagated signal coverage has become very imperative. Generally, a number of factors may impacts the level and quality of propagated signals from a transmitting point source to the receiver. The factors include transmission frequency, transmission distance, transmitter antenna heights, receiver antenna heights, engineering parameters of the antenna, terrain type (e.g. urban, suburban, and rural), earth's spherical shape, the transmission medium and the different man-made/structures obstructions that appear in the transmission path $[1,2]$. The maximum distances the radio signals can attain as they travels through a multifarious environment comprising of numerous streets paths, street widths, buildings walls, building roofs, tree leaves, rain drops, etc., could varies significantly [1,3,4]. The resultant outcome of these factors is huge signal propagation loss at the mobile subscriber receiver devises.

The ability to reliably model or estimate the signal propagation loss at the mobile subscriber communication 
devices remained a major task radio frequency engineers during telecom system network planning [5, 6]. Many of such task often comes up in mathematical and computational sciences when trying to study the regression trend pattern in nonlinear empirical dataset. One key method to solving such signal regression modelling and estimation task or problems is by applying numerical based optimisation method. The Levenberg-Marquardt algorithm (LMA) is an efficient numerical based parametric machine learning and modelling algorithm with optimal, fast, and accurate convergence speed.

\section{Literature Review}

There exist ample propagation loss models in literature (e.g, Ikegami model [7], Hata model [8], Okumura model [9], Walfisch and Bertoni model[10], COST 231 Hata model [11], etc), which have been developed for point-to-point and point-to-area predictive analysis and simulation of signal attenuation losses in wireless communication systems. However, these models' efficacies are typically valid only for a target propagation environment with similar antenna arrangement and specific frequency range [12-19]. One key way to improve on signal propagation loss predictive modelling process is by means of numerical optimisation algorithms based modeling. Besides the least squares and maximum likelihood estimation approaches, one popular numerical based optimisation that is often engaged in literature for robust predictive modelling and parametric estimation technique of measurement observation is the GaussNewton (GNA) [20-22]. The Gauss-Newton algorithm implementation procedures involves the calculation of the Jacobian matrix parameter, $\mathrm{J}$ and the resolution of typical linear and nonlinear systems at each iteration. However, a key problem with this algorithm is that its convergence properties is very slow en-route to close to solution [24, 25]. A potential solution is the engagement of Levenberg-Marquardt algorithm which is a hybrid numerical optimisation based algorithm with fast and cute convergence properties during implentation.

This work seeks to employ computationally-efficient and robust Levenberg-Marquardt algorithm (LMA) [23, 24] for adaptive parametric modelling and approximation of large-scale propagation loss data sets acquired over long term evolution (LTE) radio signal propagation techniques environment. For the purpose of comparative study and performance benchmarking, the popular Gauss-Newton algorithm is also explored to model and approximated the acquired large-scale propagation loss data sets.

\section{Methodology}

This section provides the methodology adopted in this research paper. In the methodology we start by conducting signal power measurements in the targeted environments where LTE cellular broadband radio networks are deployed. This followed by computing the propagation loss values based on the measured signal power data. Specifically, as defined in equation (3), the propagation loss expresses the differences between the transmit power and received signal power. The next step involves modelling propagation loss in the log-distance form as displayed in equation (4). Following step 3 is the application of the Levenberg-Marquardt algorithm to obtain the propagation loss model parameters. The Gauss-Newton algorithm (GNA) is also engaged as a commonly used standard approach to benchmark the proposed propagation loss modeling process. By means of relevant key performance indicators in terms of root mean square error (RMSE), mean square error (MSE), mean absolute error (MAE) and maximum absolute error (MABE), the approximation modelling capability of both LMA and GNA on the empirical propagation loss data is also considered.

\subsection{Signal Power and Propagation Loss Data}

Propagation loss data employed in this paper has been obtained from measured Reference Signal Received Power (RSRP) data performed over three LTE eNodeB cell sites. The eNodeBs with heights 30, 28 and $32 \mathrm{~m}$ respectively, operate at $2.6 \mathrm{GHz}$ carrier frequency with $10 \mathrm{MHz}$ channel bandwidths. The LTE eNodeB cell sites location were selected to highly built-up residential areas, sparsely built-up residential and developing residential areas. With the aid of integrated TEMS (Teleohone mobile systems) software investigation devices which includes one Sony Ericson TEMS pocket, one Samsung TEMS pocket, GPS, TEMS equipped Dongle and a field test Hilux bus, HP-labtop RFscanner, the RSRP measurements were made at different accessible drive test routes round all three LTE eNodeBs cells. From measured RSRP $\left(\operatorname{RSRP}_{\mathrm{m}}\right)$, we have:

$$
R S R P_{m}=P_{E I R T}-P_{\text {Loss }}
$$

and

$$
P_{\mathrm{Loss}}=\frac{\alpha_{1}}{x^{-\alpha_{2}}}
$$


From equations (1) and (2), we obtain

$$
P_{\mathrm{Loss}}=P_{E I R T}-R S R P_{m}=\frac{\alpha_{1}}{x^{-\alpha_{2}}}
$$

In $\mathrm{dB}$, equation (3) leads to:

$$
P_{\text {Loss }}(\mathrm{dB})=\alpha_{1}+10 \alpha_{2} \log 10(x)
$$

where $P_{\text {Loss }}(d B)$ is the propagation loss in decibel, Node B transmission power; $\alpha_{1}$ is a constant whose value is dependent on NodeB propagation properties. $\alpha_{2}$ indicate the propagation exponent and its value is also dependent on a several factors, which includes, transmission distance $\chi$, antenna height, cell size, carrier frequency, polarization and mostly local terrain propagation characteristics features.

\subsection{Gauss-Newton Algorithm}

Given an empirical data set of $\mathrm{n}$ pairs $\left(x_{i}, y_{i}\right)$, find the parameters $\alpha$ of a model $f\left(x_{i}, \alpha\right)$ that fits the empirical data set such that the total squares of the deviations $S(\alpha)$ between the model and the dataset is minimized:

$$
\alpha \in \arg \min _{\alpha} S(\alpha)=\arg \min \sum_{i=1}^{n}\left[y_{i}-f\left(x_{i}, \alpha\right)\right]^{2}
$$

The Gauss-Newton algorithm, is a distinctive numeric minimization algorithm with an iterative application procedure [19]. To apply it, we commence by to providing some initial guess values for the parameter vector of interest $\alpha$, The Gauss-Newton algorithm only converges iteratively to the desired global minimum if the chosen initial guess values are to some degree near the final solution.

In each iteration phase, the parameter vector $\alpha$ is constantly swapped by an alternate estimate parameter such that:

$$
f\left(x_{i}, \alpha+\delta\right) \approx f\left(x_{i}, \alpha\right)+J_{i} \delta_{i}
$$

where $J_{i}$ designate the gradient of $f$ w.r.t ; it is given by:

$$
J_{i}=\frac{\partial f\left(x_{i}, \alpha\right)}{\partial \alpha}
$$

In terms of square deviation, $S(\alpha)$, first-order approximation of $f\left(x_{i}, \alpha+\delta\right)$ is given by

$$
S(\alpha+\delta) \approx \sum_{i=1}^{n}\left[y_{i}-f\left(x_{i}, \alpha\right)-J_{i} \delta_{i}\right]^{2}
$$

Equation (4) can be written vector notation form as:

$$
\begin{gathered}
S(\alpha+\delta) \approx\|y-f(\alpha)-J \delta\|^{2} \\
=[y-f(\alpha)-J \delta]^{T}[y-f(\alpha)-J \delta] \\
=[y-f(\alpha)-J \delta]^{T}\left[(y-f(\alpha)][y-f(\alpha)]^{T} J \delta-(J \delta)^{T}[y-f(\alpha)]^{T}+J^{T} \delta^{T} J \delta\right] \\
=[y-f(\alpha)]^{T}[y-f(\alpha)]-2[y-f(\alpha)]^{T} J \delta+J^{T} \delta^{T} J \delta
\end{gathered}
$$

Taking the derivative of $S(\alpha+\delta)$ in equation (12) w.r.t $\delta$ and after some simplification gives

$$
\left(J^{T} J\right) \delta=J^{T}[y-f(\alpha)]
$$


where $J$ defines the Jacobean matrix.

\subsection{Levenberg-Marguardt Algorithm}

One key advantage of the Gauss-Newton algorithm update in equation (9) above is that it can handle modeling situations with multiple free parameters to yield optimal solution. However, the algorithm performs poorly if the initial modelling free parameters selections are far from the mark.

In [23, 24], Levenberg-Marquardt algorithm (LMA) introduced a damped parameter to cater for the weakness of the Gauss-Newton. The Levenberg-Marquardt accomplished this by replacing $\mathrm{J}^{\mathrm{T}} \mathrm{J}$ term in Gauss-Newton expression of equation (13) with $J^{T} J+\mu I$, thus resulting to

$$
\left(J^{T} J+\mu I\right) \delta=J^{T}[y-f(\alpha)]
$$

The method of modifying the diagonal elements of $\left(J^{T} J\right)$ in equation (13) is known as damping, with $\mu$ being the damping term and $I$, the identity matrix. For non-linear least square problems, equation (10) was modified in [23, 25] further to give:

$$
\left(J^{T} J+\mu \operatorname{diag}\left(J^{T} J\right)\right) \delta=J^{T}[y-f(\alpha)]
$$

Similar to the Guass-Newton algorithm in (13), the LM algorithm in (14) employs a specific system modelling function, $y=f\left(x_{i}, \alpha\right)$, to solve a regression problem recursively until convergence is attained by finding or estimating the parameters, $\alpha$ that connect the independent variables, $x$, to dependent variables, $y$, in the least square error minimization sense.

The LM algorithm relies mainly on the identity matrix $I$, Hessian matrix $H$ with the Jacobian $\mathrm{J}$, and a scalar $\mu$ that adjusts the trust region in correspondence with the error gradient. The LMA peusdocode employed in this paper is given as follows:

\section{LMA peusdocode}

1. Initialise the LMA parameter $\mu$, including the $\mu_{u p}$ and $\mu_{\text {down }}$ for damping term adjustment and as well as the initial values of parameters, $\alpha$.

2. Appraise the jacobian $\mathrm{J}$ and the residual $\mathrm{r}$ at the initial guess values

3. Compute the metric, $v=J^{T} J+\mu I$ and the cost gradient, $C_{g}=\frac{1}{2} r^{2}, \nabla C_{g}=J_{T} r$

4. Appraise the new residual (error) r, at new point $\alpha_{\text {new }}=\alpha-v^{-1} \nabla C_{g}$ and new cost, $C_{g}$ at $C_{g(\text { new })}=\frac{1}{2} r_{\text {new }}^{2}$

5. If $C_{g(\text { new })} \succ C$, retract the step and raise up the damping parameter by a factor $\mu_{u p}$, that is $\mu=\mu \times \mu_{u p}$. Otherwise, accept the step if $\alpha=\alpha_{\text {new }}, r=r_{\text {new }}$ and $\mu=\frac{\mu}{\mu_{u p}}$

6. Check the convergence. If the process is yet to converge, go to step 2. Otherwise, return the attained $\alpha$ as the best LMA modelling parameters.

\section{Results and Solution}

This section provides and discuss the results attained using Levenberg-Marquardt algorithms roust propagation loss modelling for radio signal propagation environments. For the purpose of comparative performance assessment of the proposed approach, the Gauss-Newton algorithms was also employed for propagation loss modelling. To code and implement the Levenberg-Marquardt and Gauss-Newton algorithms for the propagation loss approximation modelling, we explore the MATLAB 2018a software platform. Similar to the works by [2, 14, 19, 26], engaged different performance evaluation indicators such as RMSE, MSE, MAE and MABE to ascertain the level of the proposed algorithm. Shown in tables 1 are the Log-distance propagation path loss models and their parameters gotten using Levenberg-Marquardt algorithms and the bench-marking Gauss-Newton algorithms. The graphs in figures 1 to 7 are displayed to reveal the propagation loss approximation results using Levenberg-Marqurdt and Gauss-Newton Approximation model plus their RMSE, MSE, MAE and MABE approximation accuracy values attained for urban, suburban and rural terrains. For example, in terms of MABE as shown in chart of figure 6, the developed propagation loss model using LMA method attained improved 7.73, 14.57and 10.53 values for urban, suburban and rural terrains compared to the ones developed by GNA which yielded 15.19, 16.59 and 13.05 MABE values. The improved 
approximation performance of the LMA over the GNA can be ascribed to its capacity handle multiple free parameters and attain optimum solution irrespective of the selected values of initial guess parameters.

Table 1. Log-distance propagation path loss models and their parameters acquired using Levenberg-Marquardt algorithms and the bench-marking Gauss-Newton algorithms

\begin{tabular}{|c|c|c|c|}
\hline $\begin{array}{c}\text { Propagation Loss } \\
\text { Modeling Algorithm }\end{array}$ & $\begin{array}{c}\text { Propagation } \\
\text { Terrain }\end{array}$ & Developed Log-distance propagation loss model & $\begin{array}{c}\text { Propagation Loss } \\
\text { Attenuation exponent }\end{array}$ \\
\hline \multirow{3}{*}{ Levenberg-Marquardt } & Rural & $P_{\text {Loss }}(\mathrm{dB})=86.90+26.03 \log 10(x)$ & 2.6 \\
\cline { 2 - 4 } & Suburban & $P_{\text {Loss }}(\mathrm{dB})=69.36+34.28 \log 10(x)$ & 3.4 \\
\cline { 2 - 4 } & Urban & $P_{\text {Loss }}(\mathrm{dB})=21.16+49.32 \log 10(x)$ & 4.9 \\
\hline \multirow{3}{*}{ Gauss-Newton } & Rural & $P_{\text {Loss }}(\mathrm{dB})=75.59+29.15 \log 10(x)$ & 2.9 \\
\cline { 2 - 4 } & Suburban & $P_{\text {Loss }}(\mathrm{dB})=85.36+30.73 \log 10(x)$ & 3.1 \\
\cline { 2 - 4 } & Urban & $P_{\text {Loss }}(\mathrm{dB})=76.28+27.66 \log 10(x)$ & 2.8 \\
\hline
\end{tabular}
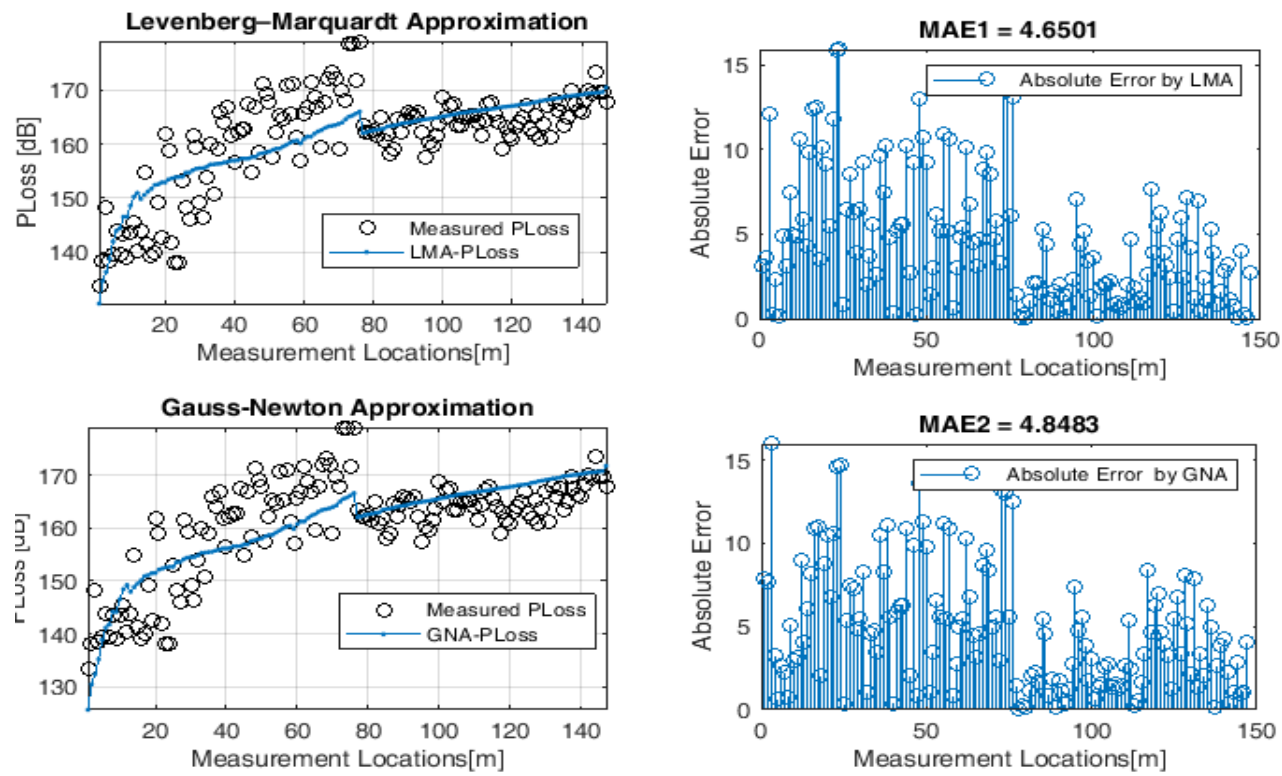

Fig.1. Propagation Loss Approximation Levenberg-Marqurdt and Gauss-Newton Approximation model plus their MAE Approximation accuracy for Urban Terrain
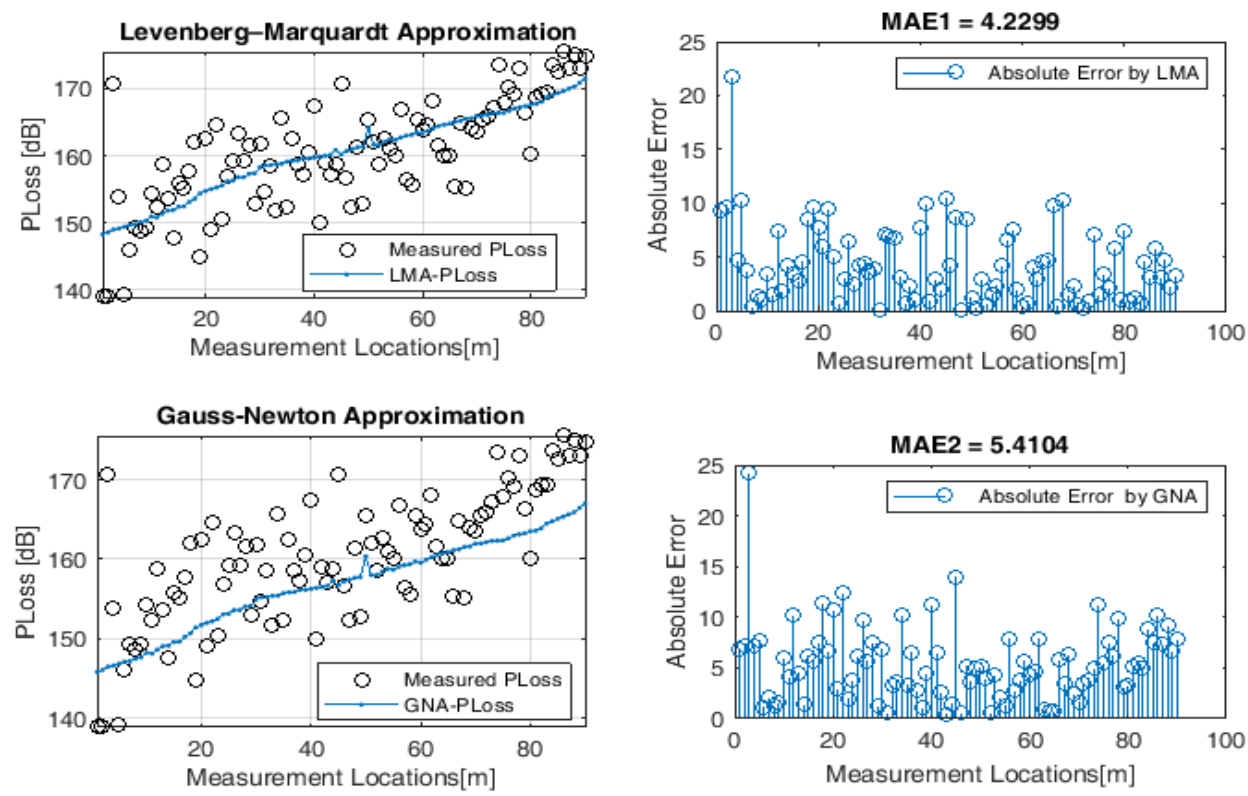

Fig.2. Propagation Loss Approximation Levenberg-Marqurdt and Gauss-Newton Approximation model plus their MAE Approximation accuracy for Suburban Terrain 

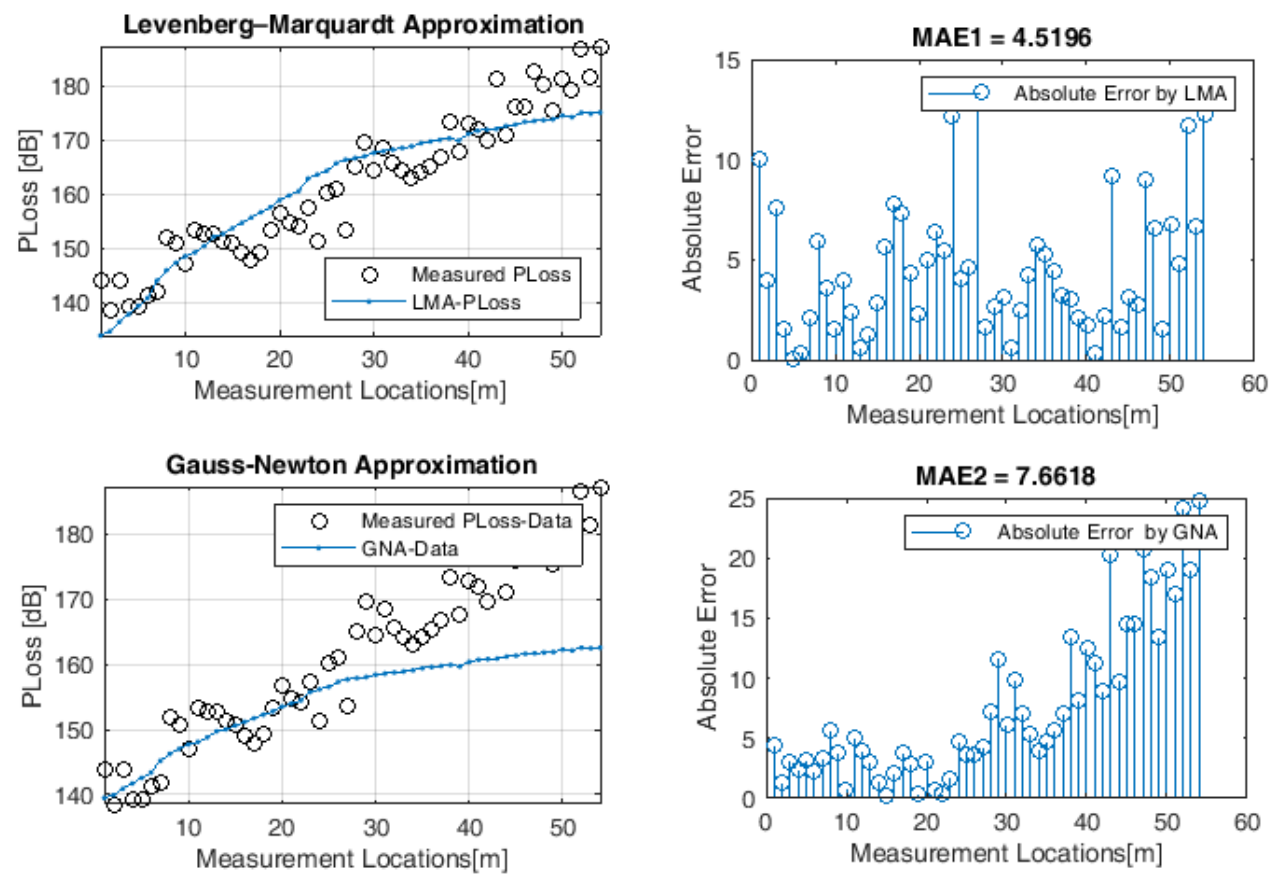

Fig.3. Propagation Loss Approximation Levenberg-Marqurdt and Gauss-Newton Approximation model plus their MAE Approximation accuracy for Rural Terrain

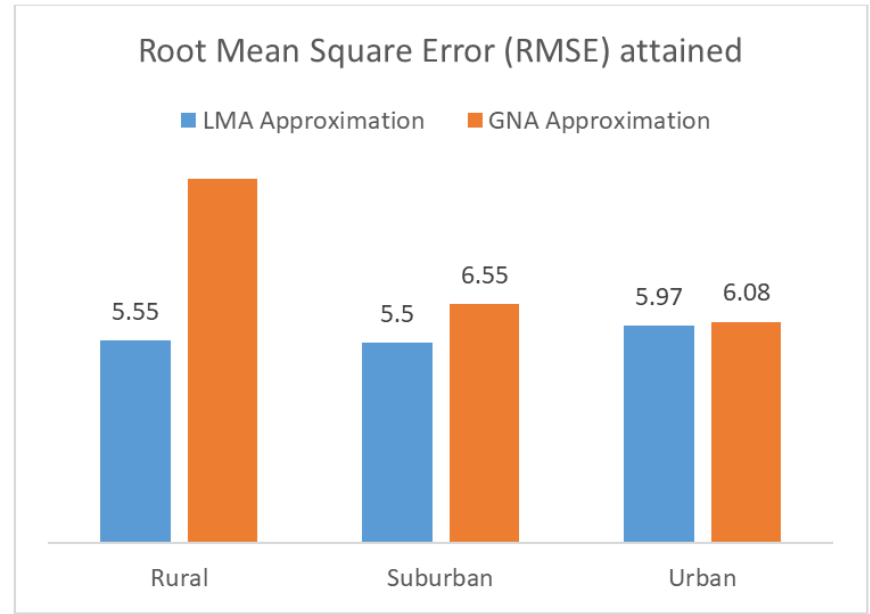

Fig.4. RMSE Propagation Loss Approximation accuracy with Levenberg-Marqurdt and Gauss-Newton Approximation model in the three Signal Propagation Terrain

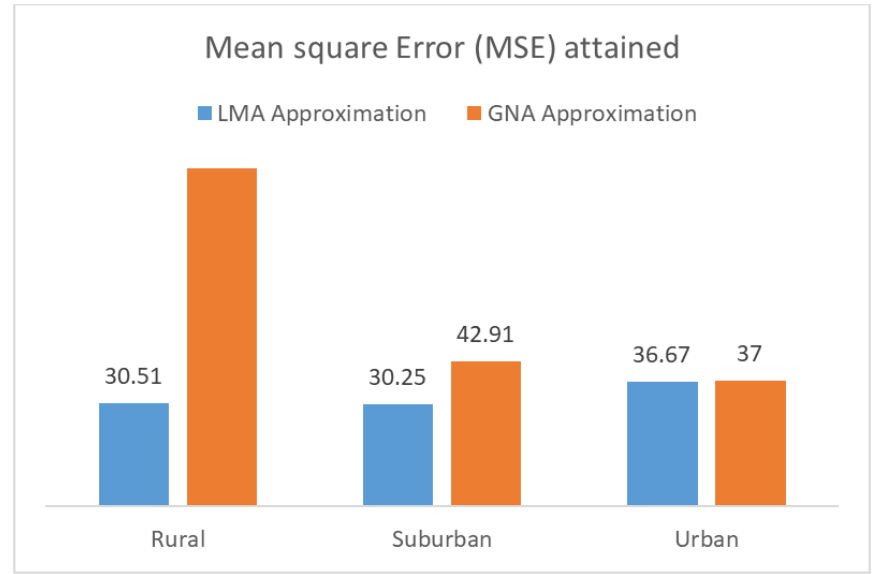

Fig.5. MSE Propagation Loss Approximation accuracy with Levenberg-Marqurdt and Gauss-Newton Approximation model in the three Signal Propagation Terrain 


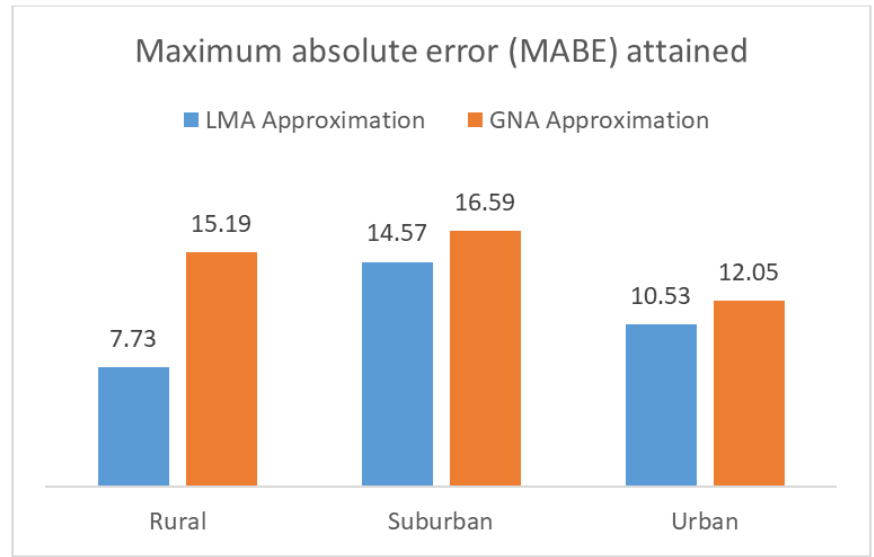

Fig.6. MABE Propagation Loss Approximation accuracy with Levenberg-Marqurdt and Gauss-Newton Approximation model in the three Signal Propagation Terrain

\section{Conclusion}

The capacity to reliably model or estimate the strength of propagated signal at the mobile subscriber communication devices remained a major task radio frequency engineers during telecom system network planning. This paper proposed and demonstrated the real-time application of the LMA to develop a log-distance propagation model based on field strength measurements.

To accomplished the above, we started by conducting signal power measurements in the targeted environments where LTE cellular broadband radio networks are deployed. The targeted LTE eNodeB propagation environments were selected to reflect typical urban, suburban and rural terrains. The next step involves modelling propagation loss in the log-distance form as displayed in equation (4). The next step was the application of the proposed Levenberg-Marquardt algorithm to a log-distance propagation model based on field strength measurements. The resultant outcome of proposed propagation loss modelling using LMA indicates more approximation modeling effectiveness compared to Gauss-Newton algorithm modelling method. Specifically, the developed propagation loss model using LMA method attained lower maximum absolute error (MABE) of 7.73, 14.57and 10.53 for urban, suburban and rural terrains compared to the ones developed by GNA which yielded 15.19, 16.59 and 13.05 MABE values. Thus, the efficacy of employing the proposed the Levenberg-Marquardt algorithm has been established.

\section{References}

[1] V.C. Ebhota, Isabona, J, and Srivastava, V.M. "Modelling, simulation and analysis of signal path loss for 4G cellular network planning", Journal of Engineering and Applied Sciences (JEAS), Vol. 13 (4), pp. 235-240.

[2] J. Isabona and D.O. Ojuh. "Adaptation of Propagation Model Parameters toward Efficient Cellular Network Planning using Robust LAD Algorithm", I.J. Wireless and Microwave Technologies, 2020, vol. 10, No.5, pp. 13-24. DOI: 10.5815/ijwmt.2020.05.02

[3] J. Isabona and G.P. Isaiah. "Computation and Verification of Propagation Loss Models based on Electric Field Data in Mobile Cellular Networks", Australian Journal of Basic and Applied Sciences, vol. 9(31), pp. 280-285, 2015.

[4] J. Isabona. "Wavelet Generalized Regression Neural Network Approach for Robust Field Strength Prediction, Wireless Personal Communication"' https://doi.org/10.1007/s11277-020-07550-5, 2020.

[5] B.S. Castro, M.R. Pinheiro, G.P. Cavalcante, I.R. Gomes, O.D.O. Carneiro. "Comparison between known propagation models using least squares tuning algorithm on $5.8 \mathrm{GHz}$ in Amazon region cities". Journal of Microwaves, Optoelectronics and Electromagnetic Applications, 10 (1), 106-113, 2011.

[6] J. Isabona and S. Azi. "Optimised Walficsh-Bertoni Model for Pathloss Prediction in Urban Propagation Environment". International Journal of Engineering and Innovative Technology (IJEIT), vol. 2 (5), pp. 14-20, 2012.

[7] F. Ikegami, and S. Yoshida. Analysis of multipath propagation structure in urban mobile radio environments. IEEE Transactions on Antenna and Propagation, vol. 28(4), pp.531-537, 1980.

[8] M. Hata, Empirical formula for propagation loss in land mobile radio services. IEEE Transactions on Vehicular Technology, vol. 29(3), pp.317-325, 1980.

[9] Y. Okumura, E. Ohmori, T. Kawano, and K. Fukuda, "Field Strength and Its Variability in VHF and UHF Land-Mobile Radio Service," Review of the Electrical Communication Laboratory, 16, pp. 825-873, September-October, 1968.

[10] J. Walfisch, and H.L. Bertoni. A theoretical model of UHF propagation in urban environments. IEEE Transactions on antennas and propagation, vol.36 (12), pp.1788-1796, 1988.

[11] COST Action231, "Digital mobile radio towards future generation system, final report,"tech. rep., European communities, EUR 18957,1999

[12] R. Mardeni and Y. P. Y. P. Lee “The Optimization of Okumura's Model for Code Division Multiple Access (CDMA) System in Malaysia'. European Journal of Scientific Research, vol.45 (4), pp.508-528, 2010. 
[13] E. AarnæS, and S. Holm. "Tuning of empirical radio propagation models effect of location accuracy”. Wireless Personal Communications, vol.30 (2-4), pp.267-281, 2004.

[14] J. Isabona and C.C. Konyeha. "Urban Area Path loss Propagation Prediction and Optimisation Using Hata Model at 800MHz". IOSR Journal of Applied Physics (IOSR-JAP), vol. 3 (4), 8-18, 2013.

[15] J. Isabona, and G.P. Isaiah. G.P. "CDMA2000 Radio Measurements at $1.9 \mathrm{GHz}$ and Comparison of Propagation Models in Three Built-Up Cities of South-South, Nigeria', American Journal of Engineering Research (AJER), Vol. 2, Issue-05, pp-96$106,2013$.

[16] J. Isabona and S.O. Azi. "Enhanced Radio Signal Loss Prediction with Correction Factors for Urban Streets in the IMT-2000 Band”, Elixir Space Science, vol. pp.15958-15962, 2013.

[17] N.N. Chimaobi, C.C. Nnadi, and A.J. Nzegwu "Comparative Study of Least Square Methods for Tuning Erceg Pathloss Model”. American Journal of Software Engineering and Applications. Vol.6 (3), pp. 61-66, 2017.

[18] Castro-Hernandez D., Paranjape R. Local Tuning of a Site-Specific Propagation Path Loss Model for Microcell Environments, International Journal of Wireless Personal communications, 91 (2), 709-728, 2016.

[19] A. Akinbolati, and O. J. Agunbiade, "Assessment of Error Bounds for Path Loss Prediction Models for TV White Space Usage in Ekiti State, Nigeria", International Journal of Information Engineering and Electronic Business(IJIEEB), Vol.12, No.3, pp. 28-39, 2020. DOI: 10.5815/ijieeb.2020.03.04.

[20] W. H. Lai, S. L. Kek and K.G. Tay" Solving Nonlinear Least Squares Problem Using Gauss-Newton Method'.IJISET International Journal of Innovative Science, Engineering \& Technology, Vol. 4 Issue 1, pp. 258-262, 2017.

[21] H.B. Nielson, “Damping Parameter In Marquardt's Method, Technical Report IMM-REP-1999-05”, Dept. of Mathematical Modeling, Technical University Denmark.

[22] A. Bjorck. Numerical methods for least squares problems, SIAM, Philadelphia, 1996.

[23] K. Levenberg. "A Method for the Solution of Certain Non-Linear Problems in Least Squares". The Quarterly of Applied Mathematics, vol. 2: 164-168 (1944).

[24] D. Marquardt, Journal of the Society for Industrial and Applied Mathematics, vol.11, pp. 431-441, 1963.

[25] W. Press, S. A. Teukolsky, W. T. Vetterling, B. P. Flannery, Numerical recipes: the art of scientific computing,, Cambridge University Press, 2007.

[26] I.B. Oluwafemi, and O. J. Femi-Jemilohun. "Propagation Profile and Strength Variation of VHF Signal in Ekiti State Nigeria", .J. Wireless and Microwave Technologies, vo.3, pp. 9-24, 2017. DOI: 10.5815/ijwmt.2017.03.02.

\section{Authors' Profiles}

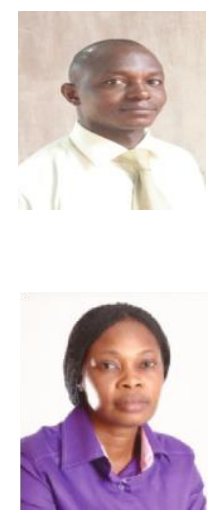

Joseph Isabona, Ph.D, received Ph.D. and M.Sc. degrees in Physics Electronics, 2013 and 2007 respectively, and a B.Sc in Applied Physics in 2003. He is the author of more than 100 scientific contributions including articles in international refereed Journals and Conferences in the area of Wireless Mobile communications. The Author is a Postdoctoral Research Fellow of the Department of Electronic Engineering, Howard College, University of KwaZulu-Natal, Durban, South Africa. His area of interest includes Signal Processing, Radio Resource Management and Physics of radio signal propagation engineering. She can be reached with josabone@ yahoo.com.

Dr. (Mrs.) O. D. Ojuh is an academic staff of Benson Idahosa University, Benin City, where she lectures Physics. She obtained her Ph.D. and M.Sc in Theoretical/ Mathematical Physics at the University of Benin City, Nigeria in 2012 and 2007 respectively and a B.Sc in Physics in 1996, from then Edo State University now known as Ambrose Alli University, Ekpoma, Edo State. Her research interest are Computational condensed matter physics/materials Science for renewable energy applications and Physics of radio signal propagation engineering. She can be reached through Email dojuh@biu.edu.ng.

How to cite this paper: Joseph Isabona, Divine O. Ojuh, "Application of Levenberg-Marguardt Algorithm for Prime Radio Propagation Wave Attenuation Modelling in Typical Urban, Suburban and Rural Terrains", International Journal of Intelligent Systems and Applications(IJISA), Vol.13, No.3, pp.35-42, 2021. DOI: 10.5815/ijisa.2021.03.04 